\title{
GENERIC VANISHING INDEX AND THE BIRATIONALITY OF THE BICANONICAL MAP OF IRREGULAR VARIETIES
}

\author{
MARTÍ LAHOZ
}

\begin{abstract}
We prove that any smooth complex projective variety with generic vanishing index bigger or equal than 2 has birational bicanonical map. Therefore, if $X$ is a smooth complex projective variety with maximal Albanese dimension and non-birational bicanonical map, then the Albanese image of $X$ is fibred by subvarieties of codimension at most 1 of an abelian subvariety of Alb $X$.
\end{abstract}

\section{INTRODUCTION}

In the study of smooth complex algebraic varieties, the natural maps provided by the holomorphic forms defined in the variety, have a special importance. For example, the invertible sheaf $\omega_{X}$ of differential $n$-forms (where $n$ is the dimension of $X$ ) produces a map to a projective space, known as the canonical map. The multiples of this canonical sheaf $\omega_{X}^{\otimes m}$ produce in this way the pluricanonical maps

$$
\varphi_{m}: X \rightarrow \mathbb{P}^{N}=\mathbb{P}\left(H^{0}\left(X, \omega_{X}^{\otimes m}\right)^{\vee}\right) .
$$

When $\varphi_{m}$ gives a birational equivalence between $X$ and its image, we will simply say that $\varphi_{m}$ is birational. We say that $X$ is of general type if for some $m>0$ the rational map $\varphi_{m}$ is birational.

For example, the curves of general type are those of genus $g \geq 2$. The tricanonical map $\varphi_{3}$ is always birational for such curves and the bicanonical $\varphi_{2}$ is also birational once that $g \geq 3$. Moreover, the canonical map is birational as soon as the curve is non-hyperelliptic.

For surfaces, Bombieri [Bo] has given sharp numerical conditions for the birationality of $\varphi_{m}$ for $m \geq 3$. The bicanonical map has revealed to be more complicated and has been studied by many algebraic geometers. In fact, the surfaces with irregularity $q(S) \leq 1$ and $\chi\left(S, \omega_{S}\right)=1$ are not completely understood and there is no classification about which ones have birational $\varphi_{2}$. For a modern review of the state of the art in the surface case, we refer to [BCP, Theorem 8].

For higher dimensions not many results are known in general. Nevertheless, the example of the bicanonical map on surfaces shows that for small irregularity $q(X)=h^{0}\left(X, \Omega_{X}^{1}\right)$, the classification becomes more difficult. For complex varieties, recall that the differential 1-forms

The author has been partially supported by the Proyecto de Investigación MTM2009-14163-C02-01. This paper was revised while the author was supported by the SFB/TR 45 'Periods, Moduli Spaces and Arithmetic of Algebraic Varieties'. 
give rise to the Albanese map

$$
\text { alb }: X \rightarrow \operatorname{Alb} X=H^{0}\left(X, \Omega_{X}^{1}\right)^{\vee} / H_{1}(X, \mathbb{Z}) .
$$

from $X$ to an abelian variety of dimension $q(X)=h^{0}\left(X, \Omega_{X}^{1}\right)$. We say that $X$ is irregular if, and only if, $\operatorname{Alb} X$ is not trivial, i.e. $q(X)>0$. And we say that $X$ is of maximal Albanese dimension (m.A.d) if, and only if, the Albanese map alb $X \rightarrow \operatorname{Alb} X$ is generically finite onto its image.

It turns out that some properties of m.A.d varieties seem to behave independently of the dimension and, indeed, Chen-Hacon showed that this is the case for their pluricanonical maps.

Theorem (Chen-Hacon. [CH2]).

(a) $X m$ m.A.d and $\chi\left(\omega_{X}\right)>0 \Rightarrow X$ is of general type, furthermore, $\varphi_{3}$ is birational.

(b) $X$ m.A.d $\Rightarrow \varphi_{6}$ is the stable pluricanonical map.

For $\varphi_{2}$, we cannot expect to use $\chi\left(\omega_{X}\right)$ to control directly its birationality. For example, if $C$ is a curve of genus 2 , then the bicanonical map of the product $C \times Y$ is never birational. In fact, it is clear that any variety that admits a fibration whose general fibre has non-birational $\varphi_{2}$ will have a non-birational bicanonical map. This should be considered, at least at first glance, as the standard case for higher dimensional varieties.

The following theorem provides geometric constraints for the non-birationality of the bicanonical map (see Theorem 5.2).

Theorem A. Let $X$ be a smooth projective complex variety of maximal Albanese dimension such that the bicanonical map is not birational. Then, the Albanese image of $X$ is fibred by subvarieties of codimension at most 1 of an abelian subvariety of $\mathrm{Alb} X$. The base of the fibration is also of maximal Albanese dimension.

That is, $X$ admits a fibration onto a normal projective variety $Y$ with $0 \leq \operatorname{dim} Y<\operatorname{dim} X$, such that any smooth model $\tilde{Y}$ of $Y$ is of maximal Albanese dimension and

$$
q(X)-\operatorname{dim} X \leq q(\tilde{Y})-\operatorname{dim} Y+1 .
$$

Hence, if $q(X)>\operatorname{dim} X+1$, the inequality implies the existence of an actual fibration, i.e. $\operatorname{dim} Y>0$, whose general fibre is mapped generically finite through the Albanese map of $X$ either onto a fixed abelian subvariety of $\operatorname{Alb} X$, or onto a divisor of this fixed abelian subvariety. When $\operatorname{dim} Y=0$ the theorem simply says that the image of $X$ in $\operatorname{Alb} X$ has codimension at most 1.

In particular, when $X$ does not admit any fibration and $q(X)>\operatorname{dim} X$, there is only one possible case, i.e. $X$ is birationally equivalent to a theta-divisor of an indecomposable principally polarized abelian variety (see [BLNP, Theorem A]). When $X$ does not admit any fibration and $q(X)=\operatorname{dim} X$, there is only one known case of variety of general type and non-birational 
bicanonical map: a double cover of a principally polarized abelian variety $(A, \Theta)$ branched along a reduced divisor $B \in|2 \Theta|$. Is this the only case? The answer is affirmative in the case of surfaces due to Ciliberto-Mendes Lopes [CM, Theorem 1.1].

To deduce Theorem A it is useful to consider the generic vanishing index introduced by Pareschi-Popa in [PP3, Definition 3.1]

$$
\operatorname{gv}\left(\omega_{X}\right)=\min _{i>0}\left\{\operatorname{codim}_{\mathrm{Pic}^{0} X} V^{i}\left(\omega_{X}\right)-i\right\},
$$

where $V^{i}\left(\omega_{X}\right)=\left\{\alpha \in \operatorname{Pic}^{0} X \mid h^{i}\left(X, \omega_{X} \otimes \alpha\right)>0\right\}$. As a consequence of Generic Vanishing Theorem of Green-Lazarsfeld [GL1, Theorem 1], we have that for any irregular variety 1 $\operatorname{dim} X \leq \operatorname{gv}\left(\omega_{X}\right) \leq q(X)-\operatorname{dim} X$.

Moreover, the negative values of $\operatorname{gv}\left(\omega_{X}\right)$ can be interpreted in terms of the dimension of the generic fibre of the Albanese map (see Theorem 3.7) and $X$ is a m.A.d variety if, and only if, $\operatorname{gv}\left(\omega_{X}\right) \geq 0$. Due to the work of Pareschi-Popa [PP3] we can interpret the positive values of $\operatorname{gv}\left(\omega_{X}\right)$ in terms of the local properties of the Fourier-Mukai transform of the structural sheaf (see Theorem 3.3). They have also proved that the positive values of $g v\left(\omega_{X}\right)$ give a lower bound for the Euler characteristic $\chi\left(\omega_{X}\right)$ (see Theorem 3.4).

Using the generic vanishing index we have the following more synthetic result.

Theorem B. Let $X$ be a smooth projective complex variety such that $\operatorname{gv}\left(\omega_{X}\right) \geq 2$. Then, the rational map associated to $\omega_{X}^{2} \otimes \alpha$ is birational onto its image for every $\alpha \in \operatorname{Pic}^{0} X$.

Theorem A is deduced from this result by an argument of Pareschi-Popa. On the other hand, this result (see Theorem 5.1) is proved using a birationality criterion (see Lemma 4.2) that is a slight modification of [BLNP, Theorem 4.13].

For curves, $\operatorname{gv}\left(\omega_{C}\right) \geq 2$ is equivalent to $g(C) \geq 3$. For surfaces, $\operatorname{gv}\left(\omega_{S}\right) \geq 2$ is equivalent to suppose that $q(S) \geq 4$ and does not admit an irregular fibration to a curve of genus $\leq q(S)-3$ (see Example 5.3).

Acknowledgments. This is part of my Ph.D. thesis. I would like to thank my advisors, Miguel Ángel Barja and Joan Carles Naranjo, for their valuable suggestions. I am also grateful to Giuseppe Pareschi for helpful conversations during my Ph.D studies. I would like to thank also Rita Pardini for her useful comments.

\section{Generalized Fourier-Mukai transform}

$X$ will be a smooth projective variety over an algebraically closed field $k$ (from section 3.3 on, we will restrict to $k=\mathbb{C}$ ). It will be equipped with a morphism $a: X \rightarrow A$ to a nontrivial abelian variety $A$, in particular, $X$ will be irregular. Let $\mathcal{P}$ be a Poincaré line bundle on $A \times \operatorname{Pic}^{0} A$. We will denote

$$
P_{a}=\left(a \times \operatorname{id}_{\operatorname{Pic}^{0} X}\right)^{*} \mathcal{P}
$$


the induced Poincaré line bundle in $X \times \operatorname{Pic}^{0} A$. When $a=$ alb, the Albanese map of $X$, then the map alb* identifies $\operatorname{Pic}^{0}(\operatorname{Alb} X)$ to $\operatorname{Pic}^{0} X$ and the line bundle $P_{\text {alb }}$ will be simply denoted by $P$.

Letting $p$ and $q$ the two projections of $X \times \operatorname{Pic}^{0} A$, we consider the left exact functor $\Phi_{P_{a}} \mathcal{F}=$ $q_{*}\left(p^{*} \mathcal{F} \otimes P_{a}\right)$, and its right derived functors

$$
R^{i} \Phi_{P_{a}} \mathcal{F}=R^{i} q_{*}\left(p^{*} \mathcal{F} \otimes P_{a}\right) .
$$

Sometimes we will have to consider the analogous derived functor $R^{i} \Phi_{P_{a}^{-1}} \mathcal{F}$ as well. By the Seesaw Theorem [Mu, Corollary 6, p. 54], $\mathcal{P}^{-1}=\left(1_{A} \times(-1)_{\operatorname{Pic}_{A} A}\right)^{*} \mathcal{P}$, so

$$
R^{i} \Phi_{P_{a}^{-1}} \mathcal{F}=\left(-1_{\mathrm{Pic}^{0} A}\right)^{*} R^{i} \Phi_{P_{a}} \mathcal{F} \quad \text { for any } i .
$$

Given a coherent sheaf $\mathcal{F}$ on $X$, its $i$-th cohomological support locus with respect to $a$ is

$$
V_{a}^{i}(\mathcal{F})=\left\{\alpha \in \operatorname{Pic}^{0} A \mid h^{i}\left(\mathcal{F} \otimes a^{*} \alpha\right)>0\right\}
$$

Again, when $a$ is the Albanese map of $X$, we will omit the subscript, simply writing $V^{i}(\mathcal{F})$. By base change, these loci contain the set-theoretical support of $R^{i} \Phi_{P_{a}} \mathcal{F}$, i.e. $\operatorname{supp} R^{i} \Phi_{P_{a}} \mathcal{F} \subseteq$ $V_{a}^{i}(\mathcal{F})$.

A way to measure the size of all the $V_{a}^{i}(\mathcal{F})$ 's is provided by the following invariant introduced by Pareschi-Popa.

Definition 2.1 ([PP3, Definition 3.1]). Given a coherent sheaf $\mathcal{F}$ on $X$, the generic vanishing index of $\mathcal{F}$ (with respect to $a$ ) is

$$
\operatorname{gv}_{a}(\mathcal{F}):=\min _{i>0}\left\{\operatorname{codim}_{\operatorname{Pic}^{0} A} V_{a}^{i}(\mathcal{F})-i\right\} .
$$

By convention we define $\operatorname{gv}_{a}(\mathcal{F})=\infty$, when $V_{a}^{i}(\mathcal{F})=\emptyset$ for every $i>0$. When $a$ is the Albanese map of $X$, we will omit the subscript, simply writing $\operatorname{gv}(\mathcal{F})$.

By base change (see [PP3, Lemma 2.1]) it is easy to see that $\operatorname{gv}_{a}(\mathcal{F})$ can be also defined as the $\min _{i>0}\left\{\operatorname{codim}_{\mathrm{Pic}^{0} A} \operatorname{supp} R^{i} \Phi_{P_{a}} \mathcal{F}-i\right\}$.

\section{Generic vanishing index of the Canonical Sheaf}

3.1. Relations between $\operatorname{gv}\left(\omega_{X}\right)$ and the Fourier-Mukai transform of $\mathcal{O}_{X}$. Here we specialize some general results of Pareschi-Popa [PP3, PP4] to the canonical sheaf of a smooth projective variety of dimension $d$. Some of these results were previously obtained by Hacon (see $[\mathrm{Ha}])$.

The negative values of the gv-index are related with the vanishing of the lowest cohomologies of the Fourier-Mukai transform of its Grothendieck dual. In the case of $\omega_{X}$ this can be stressed simply as:

Theorem 3.1 ([PP3, Theorem 2.2]). The following are equivalent, 
(a) $\operatorname{gv}_{a}\left(\omega_{X}\right) \geq-e$ for $e \geq 0$;

(b) $R^{i} \Phi_{P_{a}} \mathcal{O}_{X}=0$ for all $i \neq d-e, \ldots, d$.

Hence, when $\operatorname{gv}_{a}\left(\omega_{X}\right) \geq 0, R^{i} \Phi_{P_{a}} \mathcal{O}_{X}=0$ for all $i \neq d$, and we usually denote

$$
\widehat{\mathcal{O}_{X}}=R^{d} \Phi_{P_{a}} \mathcal{O}_{X}
$$

Note that, in this case, $H^{i}\left(X, \omega_{X} \otimes a^{*} \alpha\right)=0$ for all $i>0$ and general $\alpha \in \operatorname{Pic}^{0} A$. Therefore, by deformation-invariance of $\chi$, the generic value of $h^{0}\left(X, \omega_{X} \otimes a^{*} \alpha\right)$ equals $\chi\left(\omega_{X}\right)$, in particular $\chi\left(\omega_{X}\right) \geq 0$. Since, by base-change, the fibre of $\widehat{\mathcal{O}_{X}}$ at a general point $\alpha \in \operatorname{Pic}^{0} A$ is isomorphic to $H^{d}\left(X, a^{*} \alpha\right) \cong H^{0}\left(X, \omega_{X} \otimes a^{*} \alpha^{-1}\right)^{*}$, the (generic) rank of $\widehat{\mathcal{O}_{X}}$ is $\operatorname{rk} \widehat{\mathcal{O}_{X}}=\chi\left(\omega_{X}\right)$.

From Grothendieck-Verdier duality [Co, Theorem 4.3.1] and Theorem 3.1 it follows that,

Corollary 3.2 ([PP4, Remark 3.13]). If $\mathrm{gv}_{a}\left(\omega_{X}\right) \geq 0$ then $\mathcal{E} x t_{\mathcal{O}_{\mathrm{Pic}^{0} A}}^{i}\left(\left(-1_{\mathrm{Pic}^{0} A}\right)^{*} \widehat{\mathcal{O}_{X}}, \mathcal{O}_{\operatorname{Pic}^{0} A}\right) \cong$ $R^{i} \Phi_{P_{a}} \omega_{X}$.

The following result of Pareschi-Popa gives a dictionary between the positive values of $\operatorname{gv}_{a}\left(\omega_{X}\right)$ and the local properties of the Fourier-Mukai transform of $\widehat{\mathcal{O}_{X}}$.

Theorem 3.3 ([PP3, Corollary 3.2]). Assume that $\mathrm{gv}_{a}\left(\omega_{X}\right) \geq 0$. Then,

$$
\operatorname{gv}_{a}\left(\omega_{X}\right) \geq m \text { if, and only if, } \widehat{\mathcal{O}_{X}} \text { is a m-syzygy sheaf. }
$$

In particular, $\operatorname{gv}_{a}\left(\omega_{X}\right) \geq 1$ is equivalent to $\widehat{\mathcal{O}_{X}}$ being torsion-free and $\operatorname{gv}_{a}\left(\omega_{X}\right) \geq 2$ to $\widehat{\mathcal{O}_{X}}$ being reflexive.

Using the Evans-Griffith Syzygy Theorem and the previous theorem, Pareschi-Popa obtain the following bound on the Euler holomorphic characteristic that generalizes to higher dimensions the Castelnuovo-de Franchis inequality.

Theorem 3.4 ([PP3, Theorem 3.3]). Assume that $\mathrm{gv}_{a}\left(\omega_{X}\right) \geq 0$. Then, $\chi\left(\omega_{X}\right) \geq \mathrm{gv}_{a}\left(\omega_{X}\right)$.

Remark 3.5. In fact, the theorem of Pareschi-Popa is more general, namely that for any coherent sheaf $\mathcal{F}$ if $\infty>\operatorname{gv}_{a}(\mathcal{F}) \geq 0$, then $\chi(\mathcal{F}) \geq \operatorname{gv}_{a}(\mathcal{F})$. As a consequence, we easily obtain that for any non-zero coherent sheaf $\mathcal{F}, \operatorname{gv}_{a}(\mathcal{F}) \geq 1 \Rightarrow \chi(\mathcal{F}) \geq 1$. Observe also that if $a$ is non-trivial, we always have $\mathrm{gv}_{a}\left(\omega_{X}\right)<\infty$.

3.2. Top Fourier-Mukai transform of the canonical sheaf. In the case of abelian varieties (or complex torus) the following result is well-known and crucial in the proof of the Mukai Equivalence Theorem [M, Theorem 2.2]. We will need it in the proof of Theorem 5.1.

Proposition 3.6 ([BLNP, Proposition 6.1]). If $a^{*}: \mathrm{Pic}^{0} A \rightarrow \mathrm{Pic}^{0} X$ is an embedding, then

$$
R^{d} \Phi_{P_{a}} \omega_{X} \cong k(\hat{0})
$$


3.3. Generic vanishing theorem of Green-Lazarsfeld. The name of the gv-index comes from the well-known Generic Vanishing Theorem of Green-Lazarsfeld. As other general vanishing theorems, it requires char $k=0$ so from now on we will restrict ourselves to the case $k=\mathbb{C}$. Basically, the following theorem is [GL1, Theorem 1]. The converse implication was proven independently in [LP, Theorem B] and [BLNP, Proposition 2.9].

Theorem 3.7. For any $e>0$, the following are equivalent:

(a) the generic fibre of $a: X \rightarrow A$ has dimension $e$,

(b) $\operatorname{gv}_{a}\left(\omega_{X}\right)=-e$.

Moreover $\operatorname{gv}_{a}\left(\omega_{X}\right) \geq 0$ if, and only if, $a: X \rightarrow A$ is generically finite onto its image.

In particular, observe that for any irregular variety $1-\operatorname{dim} X \leq \operatorname{gv}\left(\omega_{X}\right) \leq q(X)-\operatorname{dim} X$.

Remark 3.8. If $\operatorname{gv}_{a}\left(\omega_{X}\right) \geq 0$ and $\chi\left(\omega_{X}\right)>0$, then $X$ is a variety of general type. Indeed, by the previous result $a: X \rightarrow A$ is generically finite and since $\chi\left(\omega_{X}\right)>0$, we have that $V_{a}^{0}\left(\omega_{X}\right)=\operatorname{Pic}^{0} A$, so by $\left[\mathrm{CH} 1\right.$, Corollary 2.4], $\kappa(X)=\operatorname{dim} X$. In particular, if $\operatorname{gv}_{a}\left(\omega_{X}\right) \geq 1$, then $X$ is of general type.

3.4. Subtorus theorem of Green-Lazarsfeld and Simpson. The following theorem is due to Green and Lazarsfeld [GL2, Theorem 0.1] with an important addition due to Simpson [S, Sections 4,6, and 7].

Theorem 3.9. Let $W$ an irreducible component of $V^{i}\left(\omega_{X}\right)$ for some $i$. Then,

(a) There exists a torsion point $\beta \in \operatorname{Pic}^{0} X$ and a subtorus $B$ of $\operatorname{Pic}^{0} X$ such that $W=\beta+B$.

(b) There exists a normal variety $Y$ of dimension $\leq d-i$, such that any smooth model of $Y$ has maximal Albanese dimension and a morphism with connected fibres $f: X \rightarrow Y$ such that $B$ is contained in $f^{*} \mathrm{Pic}^{0} Y$.

Remark 3.10. It is useful to recall that the morphism $f: X \rightarrow Y$ in the second part of the previous theorem, arises as the Stein factorization of the morphism $\pi \circ$ alb: $X \rightarrow \operatorname{Pic}^{0} W$, where $\pi:$ Alb $X \rightarrow \operatorname{Pic}^{0} W$ is the dual map of the inclusion $W \subseteq \operatorname{Pic}^{0} X$. Hence, the key point of the second part of the theorem is the dimensional bound for $Y$.

\section{Birationality CRiterion for maximal Albanese dimension varieties}

In this section, we will assume that $a: X \rightarrow A$ is a generically finite morphism onto its image, where $A$ is an abelian variety. We introduce another piece of notation.

Notation 4.1. Let $\mathcal{F}$ be a subsheaf of a line bundle and suppose that $\mathrm{gv}_{a}(\mathcal{F}) \geq 1$.

(a) We denote $U_{\mathcal{F}}$, the open subset where $h^{0}\left(\mathcal{F} \otimes a^{*} \alpha\right)$ has the minimal value, i.e. $\chi(\mathcal{F})$.

(b) Let $Z$ be the exceptional locus of $a: X \rightarrow A$, that is $Z=a^{-1}(T)$, where $T$ is the locus of points in $A$ over which the fibre of a has positive dimension. 
(c) We define

$$
\mathcal{B}_{a}^{\mathcal{F}}(x)=\left\{\alpha \in U_{\mathcal{F}} \mid x \text { is a base point of }\left|\mathcal{F} \otimes a^{*} \alpha\right|\right\} .
$$

By Remark 3.5, $\chi(\mathcal{F}) \geq 1$. So, by semicontinuity, it makes sense to speak of the base locus of $\mathcal{F} \otimes a^{*} \alpha$ for all $\alpha \in \operatorname{Pic}^{0} A$.

The following lemma is a slight modification of [BLNP, Theorem 4.13] and it is based on [PP1, Proposition 2.12 and 2.13].

Lemma 4.2. Suppose that $a: X \rightarrow A$ is a generically finite morphism onto its image and let $\mathcal{F}$ be a subsheaf of a line bundle such that $\operatorname{gv}_{a}(\mathcal{F}) \geq 1$ and $R^{i} a_{*} \mathcal{F}=0$ for all $i>0$. Suppose that for a general $x \in X$,

$$
\operatorname{codim}_{U_{\mathcal{F}}} \mathcal{B}_{a}^{\mathcal{F}}(x) \geq 2 \text {. }
$$

Then, the rational map associated to the linear system $|\mathcal{F} \otimes L|$ is birational for every line bundle $L$ such that $\operatorname{gv}_{a}(L) \geq 1$.

Proof. We first compare the Fourier-Mukai transform of $\mathcal{F} \otimes \mathcal{I}_{x}$ and $\mathcal{F}$.

Claim. Let $x \in X$ be a closed point out of $Z$. Then $R^{i} a_{*}\left(\mathcal{F} \otimes \mathcal{I}_{x} \otimes a^{*} \alpha\right)=0$ for $i>0$. This follows immediately from the exact sequence

$$
0 \rightarrow \mathcal{F} \otimes \mathcal{I}_{x} \rightarrow \mathcal{F} \rightarrow k(x) \rightarrow 0
$$

and the hypotheses that $R^{i} a_{*} \mathcal{F}=0, a$ is generically finite, and $x \notin Z$. Hence, the degeneration of the Leray spectral sequence yields to

$$
V_{a}^{i}\left(\mathcal{F} \otimes \mathcal{I}_{x}\right)=V^{i}\left(a_{*}\left(\mathcal{F} \otimes \mathcal{I}_{x}\right)\right) .
$$

By sequence (5), tensored by $a^{*} \alpha$, it follows that

$$
V_{a}^{i}\left(\mathcal{F} \otimes \mathcal{I}_{x}\right)=V_{a}^{i}(\mathcal{F}) \quad \text { for all } i \geq 2 .
$$

For $i=1$ we have the surjection $H^{1}\left(\mathcal{F} \otimes \mathcal{I}_{x} \otimes a^{*} \alpha\right) \rightarrow H^{1}\left(\mathcal{F} \otimes a^{*} \alpha\right)$, that is an isomorphism if, and only if, $x$ is not a base point of $\left|\mathcal{F} \otimes a^{*} \alpha\right|$. In other words $V_{a}^{1}\left(\mathcal{F} \otimes \mathcal{I}_{x}\right) \subseteq \mathcal{B}_{a}^{\mathcal{F}}(x) \cup V_{a}^{1}(\mathcal{F})$. Since $\operatorname{gv}_{a}(\mathcal{F}) \geq 1$, the hypothesis on $\mathcal{B}_{a}^{\mathcal{F}}(x)$ guarantees that

$$
\operatorname{codim} V_{a}^{1}\left(\mathcal{F} \otimes \mathcal{I}_{x}\right) \geq 2,
$$

for a general $x \in X \backslash Z$. Hence by $(6),(7)$ and $(8), \operatorname{gv}\left(a_{*}\left(\mathcal{F} \otimes \mathcal{I}_{x}\right)\right) \geq 1$. By [PP1, Proposition 2.13], $a_{*}\left(\mathcal{F} \otimes \mathcal{I}_{x}\right)$ is continuously globally generated (CGG, see [PP1]). Therefore $\mathcal{F} \otimes \mathcal{I}_{x}$ itself is CGG outside $Z$ (with respect to $a$ ). Since the same is true for $L$, it follows from [PP1, Proposition 2.12] that for all $\alpha \in \operatorname{Pic}^{0} A, \mathcal{F} \otimes L \otimes \mathcal{I}_{x}$ is globally generated outside $Z$. So the rational map associated to $|\mathcal{F} \otimes L|$ is birational.

Remark 4.3. From the proof we see that if $\operatorname{codim}_{U_{\mathcal{F}}} \mathcal{B}_{a}^{\mathcal{F}}(x) \geq 2$ for every $x \in X \backslash Z$, then $\mathcal{F} \otimes L$ is very ample out of $Z$, the exceptional locus of $a$. 
4.1. Adjoint line bundles. When $\mathcal{F}=\omega_{X}$ we will call $U_{\mathcal{F}}$ simply $U_{0}$ and $\mathcal{B}_{a}^{\omega_{X}}(x)$ simply by

$$
\mathcal{B}_{a}(x)=\left\{\alpha \in U_{0} \mid x \text { is a base point of } \omega_{X} \otimes a^{*} \alpha\right\} .
$$

Throughout subsections $\S 4.1$ and $\S 4.2$, we will assume that $\mathrm{gv}_{a}\left(\omega_{X}\right) \geq 1$.

Proposition-Definition 4.4. Let $X$ be a variety such that $\operatorname{gv}_{a}\left(\omega_{X}\right) \geq 1$ and let $L$ be any line bundle on $X$ such that $\operatorname{gv}_{a}(L) \geq 1$. Suppose that there exists $\alpha \in \operatorname{Pic}^{0} A$ such that $\omega_{X} \otimes L \otimes a^{*} \alpha$ is not birational. Then,

$$
\operatorname{codim}_{X \times U_{0}}\left\{(x, \alpha) \in X \times U_{0} \mid x \text { is a base point of } \omega_{X} \otimes a^{*} \alpha\right\}=1,
$$

and its divisorial part is dominant on $X$ and surjects on $U_{0}$ via the projections $p$ and $q$. We endow this set with the natural subscheme structure given by the image of the relative evaluation map $q^{*}\left(q_{*} \mathcal{L}\right) \otimes \mathcal{L}^{-1} \rightarrow \mathcal{O}_{X \times U_{0}}$, where $\left.\mathcal{L}=\left(p^{*} \omega_{X}\right) \otimes P_{a}\right)\left.\right|_{X \times U_{0}}$ and we call $\mathcal{Y}$ the union of its divisorial components that dominate $U_{0}$. Let $\overline{\mathcal{Y}}$ be its closure in $X \times \operatorname{Pic}^{0} X$. Then

(a) $X$ is covered by the scheme-theoretic fibres of the projection $\overline{\mathcal{Y}} \rightarrow U_{0}$, that we will call $F_{\alpha}$, for $\alpha$ varying in $U_{0}$. By definition, at a general point $\alpha \in U_{0}, F_{\alpha}$ is the fixed divisor of $\omega_{X} \otimes a^{*} \alpha$.

(b) For a general $x \in X$, the fibre of the projection $\overline{\mathcal{Y}} \rightarrow X$ is a divisor, that we will call $\mathcal{D}_{x}$. By definition, $\mathcal{D}_{x}$ is the closure of the union of the divisorial components of the locus of $\alpha \in U_{0}$ such that $x \in \operatorname{Bs}\left(\omega_{X} \otimes a^{*} \alpha\right)$.

Proof. Everything follows from taking $\mathcal{F}=\omega_{X}$ in Lemma 4.2. The surjectivity of the projection to $U_{0}$ is consequence of the Castelnuovo-de Franchis inequality 3.4, i.e. $\chi\left(\omega_{X}\right) \geq \operatorname{gv}_{a}\left(\omega_{X}\right) \geq$ 1.

4.2. Decomposition. In the sequel we will need $a^{*}: \operatorname{Pic}^{0} A \rightarrow \operatorname{Pic}^{0} X$ to be an embedding. However, for simplicity we will go one step further and we will simply suppose that $A=\operatorname{Alb} X$. Suppose that we are under the hypotheses of the previous Proposition-Definition and consider a fixed point $\alpha_{0} \in U_{0}$, and the map

$$
f_{\alpha_{0}}: U_{0} \rightarrow \operatorname{Pic}^{0} X \quad \alpha \mapsto \mathcal{O}_{X}\left(F_{\alpha}-F_{\alpha_{0}}\right),
$$

where $F_{\alpha}$ is the divisor defined in Proposition-Definition 4.4(a). For $\alpha \in U_{0}$, all the $F_{\alpha}$ are algebraically equivalent since they are the fibres of $\overline{\mathcal{Y}} \rightarrow U_{0}$, so the map is well-defined.

The following lemma shows that this map induces a decomposition of $\mathrm{Pic}^{0} X$ and that the divisors $F_{\alpha}$ move algebraically along a non-trivial factor of $\operatorname{Pic}^{0} X$. Although the proof is basically the same as [BLNP, Lemma 5.1], we do not require $V^{1}\left(\omega_{X}\right)$ to be a finite set, but only a proper subvariety.

Lemma 4.5. The map defined in (10), induces an homomorphism $f: \mathrm{Pic}^{0} X \rightarrow \operatorname{Pic}^{0} X$ such that, 
(a) $f^{2}=f$ and $\operatorname{Pic}^{0} X$ decomposes as $\operatorname{Pic}^{0} X \cong \operatorname{ker} f \times \operatorname{ker}(\mathrm{id}-f)$. Moreover $\operatorname{dim} \operatorname{ker}(\mathrm{id}-f)>$ 0 .

(b) Fix $\bar{\beta} \in \operatorname{ker} f$ such that $U_{0} \cap(\{\bar{\beta}\} \times \operatorname{ker}(\mathrm{id}-f))$ is non-empty. Then, for $\gamma \in U_{0} \cap$ $\operatorname{ker}(\mathrm{id}-f)$ the line bundle $\mathcal{O}_{X}\left(F_{\bar{\beta} \otimes \gamma}\right) \otimes \gamma^{-1}$ does not depend on $\gamma$. Since it is effective by semicontinuity, we call it $\mathcal{O}_{X}(F)$.

(c) For all $(\beta, \gamma) \in \operatorname{ker} f \times \operatorname{ker}(\mathrm{id}-f) \cong \operatorname{Pic}^{0} X$ such that $\beta \otimes \gamma \in U_{0},\left|\mathcal{O}_{X}(F) \otimes \gamma\right|$ is contained in the fixed divisor of $\omega_{X} \otimes \beta \otimes \gamma$.

Proof. Let $\mathcal{O}_{X}\left(M_{\alpha}\right)=\omega_{X} \otimes a^{*} \alpha \otimes \mathcal{O}_{X}\left(-F_{\alpha}\right)$. Then, the proof of $(a)$ is the same as [BLNP, Lemma 5.1](a). Item (b) follows directly from the definition of $f$. To prove $(c)$, let $(\beta, \gamma) \in$ $\operatorname{ker} f \times \operatorname{ker}(\mathrm{id}-f)$ such that $\beta \otimes \gamma \in U_{0}$ and $E \in\left|\mathcal{O}_{X}(F) \otimes \gamma\right|$. Then $\mathcal{O}_{X}\left(F_{\beta \otimes \gamma}-E\right) \cong$ $\mathcal{O}_{X}\left(F_{\beta \otimes \gamma}-F_{\bar{\beta} \otimes \gamma}\right)=f\left(\beta \otimes \bar{\beta}^{-1}\right)=\mathcal{O}_{X}$. Since $F_{\beta \otimes \gamma}$ is a fixed divisor of $\left|\omega_{X} \otimes \beta \otimes \gamma\right|$, also $E=F_{\bar{\beta} \otimes \gamma}$ is a fixed divisor in $\left|\omega_{X} \otimes \beta \otimes \gamma\right|$.

Using the decomposition given by the previous Lemma we give an explicit description of the "half" Poincaré line bundle.

Lemma 4.6 ([BLNP, Lemmas $5.1 \& 5.3])$. We call $B=\operatorname{Pic}^{0}(\operatorname{ker} f)$ and $C=\operatorname{Pic}^{0}(\operatorname{ker}(\mathrm{id}-f))$ so that

$$
\text { Alb } X \cong B \times C \quad \text { and } \quad \operatorname{Pic}^{0} X \cong \operatorname{Pic}^{0} B \times \operatorname{Pic}^{0} C,
$$

with $\operatorname{dim} C>0$. Then we have the following description of the "half" Poincaré line bundle.

$$
\left(\operatorname{alb} \times \operatorname{id}_{\operatorname{Pic}^{0} X}\right)^{*}\left(\mathcal{O}_{B \times \operatorname{Pic}^{0} B} \otimes \mathcal{P}_{C}\right) \cong \mathcal{O}_{X \times \operatorname{Pic}^{0} X}(\overline{\mathcal{Y}}) \otimes p^{*} \mathcal{O}_{X}(-F) \otimes q^{*} \mathcal{O}_{\operatorname{Pic}^{0} X}\left(-\mathcal{D}_{\bar{x}}\right),
$$

where $\bar{x}$ is such that $\operatorname{alb}(\bar{x})=0$ in $\operatorname{Alb} X$ and $\mathcal{P}_{C}$ is the Poincaré line bundle in $C \times \operatorname{Pic}^{0} C$.

Proof. The decomposition of $\operatorname{Pic}^{0} X$ comes directly from Lemma 4.5(a). By the definition of $\overline{\mathcal{Y}}$ (see Proposition-Definition 4.4) and the definition of $F$ (see Lemma 4.5(b)) we have that the line bundle

$$
\mathcal{O}_{X \times \operatorname{Pic}_{X}{ }_{X}}(\overline{\mathcal{Y}}) \otimes p^{*} \mathcal{O}_{X}(-F) \otimes q^{*} \mathcal{O}_{\operatorname{Pic}^{0} X}\left(-\mathcal{D}_{\bar{p}}\right),
$$

- restricted to $X \times\{\beta \otimes \gamma\}$ is isomorphic to $\mathcal{O}_{X}\left(F_{\beta \otimes \gamma}-F\right)=\gamma$, for all $(\beta, \gamma) \in U_{0} \subseteq$ $\operatorname{ker} f \times \operatorname{ker}(\mathrm{id}-f)$;

- restricted to $\{\bar{x}\} \times \operatorname{Pic}^{0} X$ is isomorphic to $\mathcal{O}_{\operatorname{Pic}^{0} X}\left(\mathcal{D}_{\bar{x}}\right) \otimes \mathcal{O}_{\operatorname{Pic}^{0} X}\left(-\mathcal{D}_{\bar{x}}\right)$, i.e. trivial.

On the other hand, $\left(\operatorname{alb} \times \operatorname{id}_{\operatorname{Pic}^{0} X}\right)^{*}\left(\mathcal{O}_{B \times \operatorname{Pic}^{0} B} \otimes \mathcal{P}_{C}\right)$,

- restricted to $X \times\{\beta \otimes \gamma\}$ is isomorphic to $\gamma$, for all $(\beta, \gamma) \in \operatorname{ker} f \times \operatorname{ker}(\mathrm{id}-f)$;

- restricted to $\{\bar{x}\} \times \operatorname{Pic}^{0} X$ is isomorphic to $\mathcal{O}_{\operatorname{Pic}^{0} X}$, i.e. trivial.

Then, the Lemma follows from the seesaw principle. 


\section{THE BICANONICAL MAP OF IRREGULAR VARIETIES}

The next theorem gives a sufficient numerical condition for the birationality of the bicanonical map, analogous to Pareschi-Popa Theorem [PP2, Theorem 6.1] for the tricanonical map.

Theorem 5.1. Let $X$ be a smooth projective complex variety such that $\operatorname{gv}\left(\omega_{X}\right) \geq 2$. Then, the rational map associated to $\omega_{X}^{2} \otimes \alpha$ is birational onto its image for every $\alpha \in \operatorname{Pic}^{0} X$.

As a first corollary we have the following geometric interpretation.

Theorem 5.2. Let $X$ be a smooth projective complex variety of maximal Albanese dimension such that the bicanonical map is not birational. Then $0 \leq \operatorname{gv}\left(\omega_{X}\right) \leq 1$. Moreover, it admits a fibration onto a normal projective variety $Y$ with $0 \leq \operatorname{dim} Y<\operatorname{dim} X$, any smooth model $\tilde{Y}$ of $Y$ is of maximal Albanese dimension and

$$
q(X)-\operatorname{dim} X \leq q(\tilde{Y})-\operatorname{dim} Y+\operatorname{gv}\left(\omega_{X}\right) .
$$

Proof. By Theorems 3.7 and 5.1, it is clear that $0 \leq \operatorname{gv}\left(\omega_{X}\right) \leq 1$. Now, the proof is the same as the proof of [PP3, Theorem B].

Example 5.3. We would like to show examples of varieties with $\operatorname{gv}\left(\omega_{X}\right) \geq 2$. For curves $C$, this is equivalent to $g(C) \geq 3$. For surfaces $S$, is equivalent to suppose that $q(S) \geq 4$ and $S$ does not admit an irregular fibration to a curve of genus $\leq q(S)-3$ (see [Be, Corollary 2.3]).

On the other hand, if $A$ is a simple abelian variety, then every subvariety $X$ of codimension $\geq 2$ has $\operatorname{gv}\left(\omega_{X}\right) \geq 2$. Moreover, the property of having $\operatorname{gv}\left(\omega_{X}\right) \geq 2$ is closed under taking products and cyclic coverings induced by a torsion point $\alpha \in \operatorname{Pic}^{0} X-V^{1}\left(\omega_{X}\right)$.

The rest of the paper is devoted to the proof of Theorem 5.1.

Proof. Assume that $\mathrm{gv}\left(\omega_{X}\right) \geq 1$ and there exists $\alpha \in \mathrm{Pic}^{0} X$ such that $\omega_{X}^{\otimes 2} \otimes \alpha$ is non-birational. Then, we want to see that $\operatorname{gv}\left(\omega_{X}\right)=1$. Under these hypotheses we can apply PropositionDefinition 4.4 and Lemma 4.6, so $\operatorname{Alb} X \cong B \times C$, where $B=\operatorname{Pic}^{0}(\operatorname{ker}($ id $-f))$ and $C=$ $\operatorname{Pic}^{0}(\operatorname{ker} f)$. We have the following commutative diagram

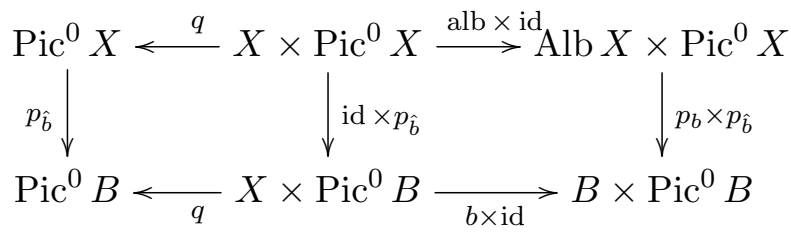

where

- $p_{b}: \operatorname{Alb} X \rightarrow B$ and $p_{\hat{b}}: \operatorname{Pic}^{0} X \rightarrow \operatorname{Pic}^{0} B$ are the corresponding projections,

- $b$ is the composition by $b: X \stackrel{\text { alb }}{\rightarrow}$ Alb $X \stackrel{p_{b}}{\rightarrow} B$, and

- abusing notation we also call $q$ either the projection $X \times \operatorname{Pic}^{0} X \rightarrow \operatorname{Pic}^{0} X$ or $X \times$ $\mathrm{Pic}^{0} B \rightarrow \mathrm{Pic}^{0} B$ and $p$ the projections $X \times \mathrm{Pic}^{0} X \rightarrow X$ or $X \times \mathrm{Pic}^{0} B \rightarrow X$. 
The effectiveness of $\overline{\mathcal{Y}}$ give us the following short exact sequence on $X \times \operatorname{Pic}^{0} X$

$$
\left.0 \rightarrow(\mathrm{alb} \times \mathrm{id})^{*}\left(\mathcal{O}_{B \times \mathrm{Pic}^{0} B} \otimes \mathcal{P}_{C}\right)^{-1} \stackrel{\overline{\mathcal{Y}}}{\rightarrow} p^{*} \mathcal{O}_{X}(F) \otimes q^{*} \mathcal{O}\left(\mathcal{D}_{\bar{x}}\right) \rightarrow\left(p^{*} \mathcal{O}_{X}(F) \otimes q^{*} \mathcal{O}\left(\mathcal{D}_{\bar{x}}\right)\right)\right|_{\overline{\mathcal{Y}}} \rightarrow 0 .
$$

Recall that $P=\left(\operatorname{alb} \times \operatorname{id}_{\operatorname{Pic}^{0} X}\right)^{*}\left(\mathcal{P}_{B} \otimes \mathcal{P}_{C}\right)$ since the Poincaré line bundle $\mathcal{P}$ in Alb $X \times \operatorname{Pic}^{0} X$ is isomorphic to $\mathcal{P}_{B} \otimes \mathcal{P}_{C}$. We apply the functor $R^{d} q_{*}\left(\cdot \otimes\left(\operatorname{alb} \times \operatorname{id}_{\mathrm{Pic}^{0} X}\right)^{*}\left(\mathcal{P}_{B}^{-1} \otimes \mathcal{O}_{C \times \mathrm{Pic}^{0} C}\right)\right)$, that is, we tensor by the other "half" Poincaré line bundle and we consider the top direct image. We get

$$
\begin{aligned}
\cdots & \rightarrow R^{d} \Phi_{P^{-1}}\left(\mathcal{O}_{X}\right) \rightarrow R^{d} q_{*}\left(p^{*} \mathcal{O}_{X}(F) \otimes\left(\operatorname{alb} \times \operatorname{id}_{\operatorname{Pic}^{0} X}\right)^{*}\left(\mathcal{P}_{B}^{-1} \otimes \mathcal{O}_{C \times \operatorname{Pic}^{0} C}\right)\right) \otimes \mathcal{O}_{\operatorname{Pic}^{0} X}\left(\mathcal{D}_{\bar{x}}\right) \rightarrow \\
& \rightarrow R^{d} q_{*}\left(\left.\left(p^{*} \mathcal{O}_{X}(F) \otimes q^{*} \mathcal{O}_{\operatorname{Pic}^{0} X}\left(\mathcal{D}_{\bar{x}}\right)\right)\right|_{\bar{Y}} \otimes\left(\operatorname{alb} \times \operatorname{id}_{\operatorname{Pic}^{0} X}\right)^{*}\left(\mathcal{P}_{B}^{-1} \otimes \mathcal{O}_{C \times \operatorname{Pic}^{0} C}\right)\right) \rightarrow 0
\end{aligned}
$$

Using that $R^{i} \Phi_{P^{-1}} \cong(-1)_{\mathrm{Pic}^{0} X}^{*} R^{i} \Phi_{P}$ (see (3)), we have the following short exact sequence,

$$
0 \rightarrow(-1)_{\mathrm{Pic}^{0} X}^{*} \widehat{\mathcal{O}_{X}} \stackrel{\mu}{\rightarrow} \mathcal{E}\left(\mathcal{D}_{\bar{x}}\right) \rightarrow \mathcal{T} \rightarrow 0
$$

where:

(a) By base change, $\mathcal{E}=R^{d} q_{*}\left(p^{*} \mathcal{O}_{X}(F) \otimes\left(\operatorname{alb} \times \operatorname{id}_{\mathrm{Pic}^{0} X}\right)^{*}\left(\mathcal{P}_{B}^{-1} \otimes \mathcal{O}_{C \times \operatorname{Pic}^{0} C}\right)\right)$ is a coherent sheaf of rank $h^{d}\left(\mathcal{O}_{X}(F) \otimes \beta^{-1}\right)$ by a general $\beta \in \operatorname{ker} f$, i.e $h^{0}\left(\omega_{X} \otimes \mathcal{O}_{X}(-F) \otimes \beta\right)=\chi\left(\omega_{X}\right)$ by Lemma $4.5(c)$. Then,

$$
\begin{array}{rlr}
\mathcal{E} & =R^{d} q_{*}\left(p^{*} \mathcal{O}_{X}(F) \otimes(\mathrm{alb} \times \mathrm{id})^{*}\left(\mathcal{P}_{B}^{-1} \otimes \mathcal{O}_{C \times \mathrm{Pic}^{0} C}\right)\right) & \\
& =R^{d} q_{*}\left(p^{*} \mathcal{O}_{X}(F) \otimes\left(\mathrm{id} \times p_{\hat{b}}\right)^{*}(b \times \mathrm{id})^{*} \mathcal{P}_{B}^{-1}\right) & \text { right square of }(11) \\
& =R^{d} q_{*}\left(\mathrm{id} \times p_{\hat{b}}\right)^{*}\left(p^{*} \mathcal{O}_{X}(F) \otimes(b \times \mathrm{id})^{*} \mathcal{P}_{B}^{-1}\right) & \text { abuse of notation on } p \\
& =p_{\hat{b}}^{*} R^{d} q_{*}\left(p^{*} \mathcal{O}_{X}(F) \otimes(b \times \mathrm{id})^{*} \mathcal{P}_{B}^{-1}\right) & \text { flat base change } \\
& =p_{\hat{b}}^{*} R^{d} \Phi_{P_{b}^{-1}}\left(\mathcal{O}_{X}(F)\right), &
\end{array}
$$

following the notation of (1) and (2).

(b) $\mathcal{T}=R^{d} q_{*}\left(\left.\left(p^{*} \mathcal{O}_{X}(F) \otimes q^{*} \mathcal{O}_{\operatorname{Pic}^{0} X}\left(\mathcal{D}_{\bar{x}}\right)\right)\right|_{\bar{y}} \otimes\left(\operatorname{alb} \times \operatorname{id}_{\mathrm{Pic}^{0} X}\right)^{*}\left(\mathcal{P}_{B}^{-1} \otimes \mathcal{O}_{C \times \mathrm{Pic}^{0} C}\right)\right)$ is supported at the locus of the $\alpha \in \operatorname{Pic}^{0} X$ such that the fibre of the projection $q: \overline{\mathcal{Y}} \rightarrow \operatorname{Pic}^{0} X$ has dimension $d$, i.e. it coincides with $X$. Such locus is contained in $V^{1}\left(\omega_{X}\right)$, therefore, since $\operatorname{gv}\left(\omega_{X}\right) \geq 1, \operatorname{codim} \operatorname{supp} \mathcal{T} \geq 2$.

(c) The map $\mu$ is injective since it is a generically surjective map of sheaves of the same rank (recall that $\operatorname{rk} \widehat{\mathcal{O}_{X}}=\chi\left(\omega_{X}\right)$ ), and, as $\operatorname{gv}\left(\omega_{X}\right) \geq 1$, the source $\widehat{\mathcal{O}_{X}}$ is torsion-free (Theorem 3.3).

(d) $\mu$ is $R^{d} q_{*}\left(m_{s}\right)$, where $m_{s}$ is the multiplication by the section defining $\overline{\mathcal{Y}}$. By base change [Mu, Corollary 3, p. 53], $R^{d} q_{*}\left(m_{s}\right) \otimes \mathbb{C}(\alpha)=H^{d}\left(\left.m_{s}\right|_{q^{-1}\{\alpha\}}\right)$ where $q$ is the projection $q: \overline{\mathcal{Y}} \rightarrow \operatorname{Pic}^{0} X$. When $q^{-1}\{\alpha\}=X,\left.m_{s}\right|_{q^{-1}\{\alpha\}}=0$, so in these points $R^{d} q_{*}\left(m_{s}\right) \otimes \mathbb{C}(\alpha)=0$.

Claim 5.4. $\mathcal{T} \neq 0$. 
Proof of the Claim. Suppose that $\mathcal{T}=0$, so $\mu$ is an isomorphism. Taking $\mathcal{E} x t^{d}\left(\cdot, \mathcal{O}_{\operatorname{Pic}^{0} X}\right)$ we get

$$
\begin{aligned}
& k(\hat{0})=R^{d} \Phi_{P} \omega_{X} \\
& \text { Proposition } 3.6 \\
& =\mathcal{E} x t^{d}\left(\mathcal{E}, \mathcal{O}_{\operatorname{Pic}^{0} X}\right) \otimes \mathcal{O}\left(-\mathcal{D}_{\bar{x}}\right) \\
& =p_{\hat{b}}^{*} \mathcal{E} x t^{d}\left(R^{d} \Phi_{P_{b}}\left(\mathcal{O}_{X}(F)\right), \mathcal{O}_{\operatorname{Pic}^{0} B}\right) \otimes \mathcal{O}\left(-\mathcal{D}_{\bar{x}}\right) \\
& \begin{array}{r}
\mathcal{E} x t^{d}\left(\mu, \mathcal{O}_{\text {Pic }^{0} X}\right) \text { and Cor. } 3.2 \\
\text { see item (a), }
\end{array}
\end{aligned}
$$

which implies that $\operatorname{codim}_{\operatorname{Alb} X} B=\operatorname{dim} \operatorname{ker}(\mathrm{id}-f)=0$ contradicting Lemma 4.6.

Let $\tau\left(\mathcal{E}\left(\mathcal{D}_{\bar{x}}\right)\right)$ be the torsion part of $\mathcal{E}\left(\mathcal{D}_{\bar{x}}\right)$ and $\widetilde{\mathcal{E}\left(\mathcal{D}_{\bar{x}}\right)}$ the quotient of $\mathcal{E}\left(\mathcal{D}_{\bar{x}}\right)$ by its torsion part. Hence $\widehat{\mathcal{E}\left(\mathcal{D}_{\bar{x}}\right)}$ is torsion-free. Now consider the following composition

$$
\begin{aligned}
& (-1)_{\mathrm{Pic}^{0} X}^{*} \widehat{\mathcal{O}_{X}} \stackrel{\mu}{\longrightarrow} \mathcal{E}\left(\mathcal{D}_{\bar{x}}\right) \\
& \widetilde{\tilde{\mu}} \underset{\mathcal{E}\left(\mathcal{D}_{\bar{x}}\right) .}{\Downarrow}
\end{aligned}
$$

Since $\tilde{\mu}$ is generically surjective and $(-1)_{\operatorname{Pic}^{0} X}^{*} \widehat{\mathcal{O}_{X}}$ is torsion-free (recall that $\operatorname{gv}\left(\omega_{X}\right) \geq 1$ ), we have that $\tilde{\mu}$ is injective. Completing the diagram we get,

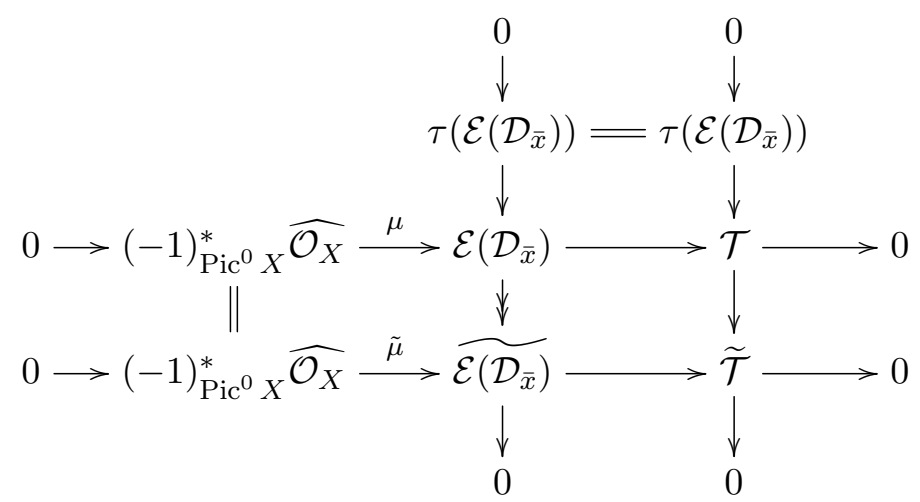

If $\widetilde{\mathcal{T}}=0$, then the middle horizontal short exact sequence splits. But, for $\alpha$ a closed point in the support of $\mathcal{T}$ (by the previous claim we know that $\mathcal{T} \neq 0$ ), $\mu \otimes \mathbb{C}(\alpha)=0$ by item (d), so $\mu$ cannot split. Therefore $\tilde{\mathcal{T}} \neq 0$.

Let $e=\operatorname{codim}_{\operatorname{Pic}^{0} X} \operatorname{supp} \widetilde{\mathcal{T}} \geq 2($ see item $(c))$. Then $\operatorname{codim}_{\operatorname{Pic}^{0} X} \operatorname{supp} \mathcal{E} x t^{e}\left(\widetilde{\mathcal{T}}, \mathcal{O}_{\operatorname{Pic}^{0} X}\right)=e$. Now, we apply the functor $\mathcal{E} x t^{i}\left(\cdot, \mathcal{O}_{\mathrm{Pic}^{0} X}\right)$ to the bottom row of (13) using Corollary 3.2

$$
\ldots \rightarrow R^{e-1} \Phi_{P} \omega_{X} \rightarrow \mathcal{E} x t^{e}\left(\tilde{\mathcal{T}}, \mathcal{O}_{\operatorname{Pic}^{0} X}\right) \rightarrow \mathcal{E} x t^{e}\left(\widetilde{\mathcal{E}\left(\mathcal{D}_{\bar{x}}\right)}, \mathcal{O}_{\operatorname{Pic}^{0} X}\right) \rightarrow \ldots
$$

Since $\widetilde{\mathcal{E}\left(\mathcal{D}_{\bar{x}}\right)}$ is torsion-free, $\operatorname{codim}_{\mathrm{Pic}^{0} X} \operatorname{supp} \mathcal{E} x t^{e}\left(\widetilde{\mathcal{E}\left(\mathcal{D}_{\bar{x}}\right)}, \mathcal{O}_{\mathrm{Pic}^{0} X}\right)>e$. Therefore, we must have $\operatorname{codim}_{\mathrm{Pic}^{0} X} \operatorname{supp} R^{e-1} \Phi_{P} \omega_{X}=e$ and $\operatorname{gv}\left(\omega_{X}\right) \leq 1$. 


\section{REFERENCES}

[BLNP] M.A. Barja, M. Lahoz, J.C. Naranjo and G. Pareschi, On the bicanonical map of irregular varieties, preprint arXiv:0907.4363. To appear in J. Algebraic Geom.

[BCP] I. C. Bauer, F. Catanese, and R. Pignatelli. Complex surfaces of general type: some recent progress. In Global aspects of complex geometry, pages 1-58. Springer, Berlin, 2006.

[Be] A. Beauville. Annulation du $H^{1}$ pour les fibrés en droites plats. In Complex algebraic varieties (Proc. Bayreuth 1990), pages 1-15; Springer, Berlin, 1992.

[Bo] E. Bombieri. Canonical models of surfaces of general type. Inst. Hautes Études Sci. Publ. Math., (42):171219, 1973.

[CH1] J. A. Chen and C. D. Hacon. Pluricanonical maps of varieties of maximal Albanese dimension. Math. Ann., 320(2):367-380, 2001.

[CH2] Linear series of irregular varieties. In Algebraic geometry in East Asia (Kyoto, 2001), pages 143-153. World Sci. Publ., River Edge, NJ, 2002.

[CM] C. Ciliberto and M. Mendes Lopes. On surfaces with $p_{g}=q=2$ and non-birational bicanonical maps. Adv. Geom., 2(3):281-300, 2002.

[Co] B. Conrad. Grothendieck duality and base change, volume 1750 of Lecture Notes in Mathematics. Springer-Verlag, Berlin, 2000.

[GL1] M. Green and R. Lazarsfeld. Deformation theory, generic vanishing theorems, and some conjectures of Enriques, Catanese and Beauville. Invent. Math., 90(2):389-407, 1987.

[GL2] Higher obstructions to deforming cohomology groups of line bundles. J. Amer. Math. Soc., 4(1):87-103, 1991.

[Ha] C. D. Hacon. A derived category approach to generic vanishing. J. Reine Angew. Math., 575:173-187, 2004.

[LP] R. Lazarsfeld and M. Popa. Derivative complex, BGG correspondence, and numerical inequalities for compact Kaehler manifolds. Invent. Math., 182 no.3 (2010), 605-633.

[M] S. Mukai. Duality between $D(X)$ and $D(\hat{X})$ with its application to Picard sheaves. Nagoya Math. J., 81:153-175, 1981.

[Mu D. Mumford. Abelian varieties, volume 5 of Tata Institute of Fundamental Research Studies in Mathematics. Published for the Tata Institute of Fundamental Research, Bombay, 2008. With appendices by C. P. Ramanujam and Yuri Manin, Corrected reprint of the second (1974) edition.

[PP1] G. Pareschi and M. Popa. Regularity on abelian varieties I. J. Amer. Math. Soc., 16(2):285-302, 2003.

[PP2] Regularity on abelian varieties III: relationship with generic vanishing and applications. Clay Math. Proc., 2006.

[PP3] Strong generic vanishing and a higher-dimensional Castelnuovo-de Franchis inequality. Duke Math. J., 150(2):269-285, 2009.

[PP4] GV - G - Gheaves, Fourier-Mukai transform, and Generic Vanishing. Amer. J. Math., 133, 235-271, 2011.

[S] C. Simpson. Subspaces of moduli spaces of rank one local systems. Ann. Sci. École Norm. Sup. (4), 26(3):361-401, 1993.

Departament d’Àlgebra i Geometria, Facultat de Matemàtiques, Universitat de Barcelona. Gran Via, 585, 08007 BARCElona. Spain

E-mail address: marti.lahoz@ub.edu

Current address: Mathematisches Institut, Universität Bonn, Endenicher Allee 60, 53115 Bonn, Germany. 